\title{
CARACTERIZAÇÃO DE ROCHAS RESERVATÓRIO POR MICROTOMOGRAFIA DE RAIOS X
}

\author{
L. PALOMBO, C. ULSEN ${ }^{*}$, D. ULIANA, F.R. COSTA, M. YAMAMOTO e H. KAHN \\ Universidade de São Paulo (USP), Departamento de Engenharia de Minas e de Petróleo, \\ Laboratório de Caracterização Tecnológica, Laboratório de Mecânica de Rochas \\ carina@Ict.poli.usp.br
}

Artigo submetido em junho/2015 e aceito em setembro/2015

DOI: 10.15628/holos.2015.3103

\section{RESUMO}

Reservatórios de petróleo são rochas sedimentares com porosidade e permeabilidade suficientes para armazenar e permitir o fluxo de hidrocarbonetos. Testemunhos das rochas reservatório são coletados para a caracterização e obtenção de dados petrofísicos como, por exemplo, porosidade e permeabilidade. Atualmente, a caracterização de rochas reservatório envolve diversas análises como microscopia óptica e/ou eletrônica, medição de porosidade e permeabilidade através de injeção de gás nitrogênio, etc. Estas análises, apesar de consagradas, consomem muito tempo, principalmente, para a preparação das amostras retiradas dos testemunhos. Neste cenário, a aplicação da microtomografia de raios-X (MRX), por meio da aquisição e processamento de imagens tridimensionais na escala dos poros da amostra, em geral demandando
\end{abstract}

pouca ou nenhuma preparação, permite a criação de um modelo digital tridimensional da rede de poros e associações minerais para o estudo das fácies litológicas e suas microestruturas. Este estudo propõe um procedimento para aquisição e processamento de imagens tridimensionais de amostras de rochas reservatório obtidas por MRX. A natureza 3D dos dados obtidos proporciona novas perspectivas para a avaliação da heterogeneidade das rochas e simulação do fluxo no meio poroso. A partir da análise digital de rochas, novos estudos poderão ser realizados aplicando-se a MRX como uma ferramenta para estudos de investigação sedimentológica/diagenética, caracterização da rocha reservatório e análise/modelagem de porosidade e permeabilidade.

PALAVRAS-CHAVE: microtomografia de raios $X$, rocha reservatório, porosidade, permeabilidade, petrofísica.

\section{RESERVOIR ROCK CHARACTERIZATION BY X-RAY MICROTOMOGRAPHY}

\begin{abstract}
Petroleum reservoirs are sedimentary rocks with sufficient porosity and permeability to store and allow hydrocarbon flow. Testimonies of reservoir rocks are collected to be characterize and to obtain petrophysical data, for example, porosity and permeability. Currently, the characterization of reservoir rocks involves various analyzes such as optical and/or electron microscopy, porosity and permeability measurement using nitrogen gas injection, etc. These analyzes, although dedicated, time consuming, especially for the preparation of samples of the testimonies. In this scenario, the application of X-ray microtomography (MRX) by acquiring and processing three-dimensional images on the scale of the pores of the sample, usually requiring
\end{abstract}

little or no preparation, allows the creation of a threedimensional digital model of the network pores and minerals associations for the study of lithological facies and their microstructures. This study proposes a procedure for acquiring and processing threedimensional images of reservoir rock samples obtained by MRX. The 3D nature of the data provides new perspectives for the evaluation of heterogeneity of rocks and simulation of flow in porous media. From the digital analysis of rocks, further studies can be carried out by applying the MRX as a tool for research studies sedimentological/diagenetic characterization of reservoir rock and analysis/modeling porosity and permeability.

KEYWORDS: X-ray microtomography, reservoir rock, porosity, permeability, petrophysics. 


\section{INTRODUÇÃO}

Um projeto de Exploração \& Produção possui várias fases, a primeira fase é chamada de "Exploração" quando os estudos geológicos e métodos geofísicos determinam as formações da bacia sedimentar com potencial de acumulação de hidrocarbonetos. Então um poço exploratório pioneiro é perfurado e através das análises do cascalho proveniente da perfuração (mud logging), medições com ferramentas de perfilagem geofísica, teste de produção da formação e análise de testemunhos confirmam o acúmulo de hidrocarbonetos nas formações alvos. Se confirmado a existência de acúmulo de hidrocarboneto, a fase de "Avaliação" é iniciada verificando conectividade do reservatório.

Ainda durante a avaliação é realizado a modelagem do reservatório que utiliza dados geofísicos de sísmica e dados colhidos de todos os poços exploratórios para determinar o volume da rocha reservatório. Com a informação do volume da rocha reservatório e os dados petrofísicos provenientes da caracterização das amostras da rocha reservatório como por exemplo, a porosidade, é possível estimar o volume de hidrocarbonetos dentro do reservatório. A fase de Avaliação se encerra com a simulação de fluxo do reservatório. A simulação necessita de outros dados da caracterização das amostras, como a permeabilidade, para determinar a localização dos poços de desenvolvimento, além de calcular as curvas de produção do campo que determina se o projeto é economicamente viável.

O breve relato acima demonstra que a caracterização das rochas reservatório torna-se componente fundamental em um projeto de exploração e produção de hidrocarbonetos, uma vez que as informações obtidas embasarão decisões críticas ao projeto. As análises petrofísicas tradicionais possuem rotinas complexas, como por exemplo, a limpeza e remoção do fluido saturante de dentro da amostra do testemunho, que podem levar meses.

A técnica de microtomografia de raios $X(M R X)$ na caracterização de rochas reservatório vem aprofundar o estudo de reservatórios petrolíferos e depósitos minerais. Com uma metodologia mais simples na preparação de amostras e geração de imagens 3D, a MRX possibilita que informações fundamentais como porosidade e permeabilidade sejam aferidas com maior rapidez e precisão, além de ser um método não-destrutivo. Neste sentido, a caracterização por MRX proporciona uma aquisição direta de dados volumétricos, análise quantitativa em 3D, menor tempo de análise, etc. (MILLER e LIN, 2004).

A técnica baseia-se no princípio de geração de imagens por raios $X$, no qual várias radiografias do objeto analisado são registradas em diferentes ângulos de rotação. Tais imagens são utilizadas para compor uma representação em três dimensões através de processamento computacional. A amostra é posicionada entre a fonte de emissão de raios $\mathrm{X}$ e o detector, sendo então rotacionada para a aquisição de projeções em várias posições. Essas projeções são proporcionais à quantidade de radiação que chega ao detector, que varia de acordo com os distintos coeficientes de atenuação dos raios X de cada material (LIN, L. et al., 2002).

A tomografia computadorizada de alta resolução possibilita a visualização e caracterização da estrutura interna de objetos em três dimensões. Como é uma técnica de rápida aquisição de dados tridimensionais e não requer nenhum preparo com maior rigor e delicadeza (como limpeza de rochas contendo hidrocarbonetos, impregnação, desbaste, polimento, etc.), é frequentemente usada em testes qualitativos e quantitativos de pesquisa geológica. Além disso, 
- método fornece dados numéricos, que podem conduzir sistematicamente a conclusões objetivas (DULIU, 1999 e MEES, SWENNEN et al. 2003).

O presente trabalho apresenta as possibilidades da MRX no estudo de rochas análogas a rochas reservatório, quanto à caracterização da microestrutura interna, quantificação de espaço poroso e conectividade.

\section{MATERIAIS E MÉTODOS}

O presente estudo foi realizado em amostras de afloramento comercializadas como análogos de rochas reservatório. Foram caracterizadas duas amostras de arenito e duas de calcário, referentes a afloramentos norte-americanos descritos na Tabela 1.

Tabela 1 - Relação de amostras estudadas

\begin{tabular}{lll}
\hline Amostra & Formação & Tipo de rocha \\
\hline BS - Bentheimer Sandstone & Germany & Arenito \\
GBS - Grey Berea Sandstone & Kipton & Arenito \\
DPL - Desert Pink Limestone & Edwards Plateau & Calcário \\
IL - Indiana Limestone & Bedford & Calcário \\
\hline
\end{tabular}

Todas as amostras apresentam feições homogêneas e isotrópicas. O procedimento empregado na caracterização das amostras envolveu a determinação dos parâmetros de porosidade por análise digital em imagens 3D obtidas por MRX em equipamento Zeiss, modelo Xradia VersaXRM-510.

Os corpos-de-prova (um para cada amostra) foram obtidos a partir de testemunhos de rocha cilíndricos, com 15 a $20 \mathrm{~cm}$ de altura. Inicialmente, os corpos-de-prova foram cortados com serra de disco diamantado para obtenção de uma pastilha com $20 \mathrm{~mm}$ de altura; na sequência, foi extraída uma nova amostra cilíndrica de $10 \mathrm{~mm}$ de diâmetro por $20 \mathrm{~mm}$ de altura por meio de corte com jato de água de alta pressão.

Para a aquisição das imagens de MRX, padronizaram-se os seguintes parâmetros: energia do feixe em $80 \mathrm{kV}$ com potência de $7 \mathrm{~W}$, tempo de exposição de 7 segundos por projeção e tempo de coleta de $2 \mathrm{~h} 15 \mathrm{~min}$, filtro LH6 (Low Energy), tamanho do pixel de $4 \mu \mathrm{m}$ e campo de visão de $4 \mathrm{~mm}^{2}$. As imagens 3D reconstruídas foram processadas de modo a caracterizar a microestrutura interna das amostras ressaltando porosidade total e conectividade dos poros. As imagens reconstruídas em 3 dimensões foram processadas utilizando-se o software Avizo Fire 9.0 (FEI). O processamento das imagens contemplou a aplicação de filtro digital para redução de ruído, segmentação dos espaços vazios para posterior cálculo da porosidade em função da proporção do volume de poros pelo volume total.

\section{RESULTADOS E DISCUSSÃO}

O processo de segmentação em corte bidimensional de cada amostra e a representação do volume de dados tratados digitalmente é mostrado nas figuras de 1 a 4 . 


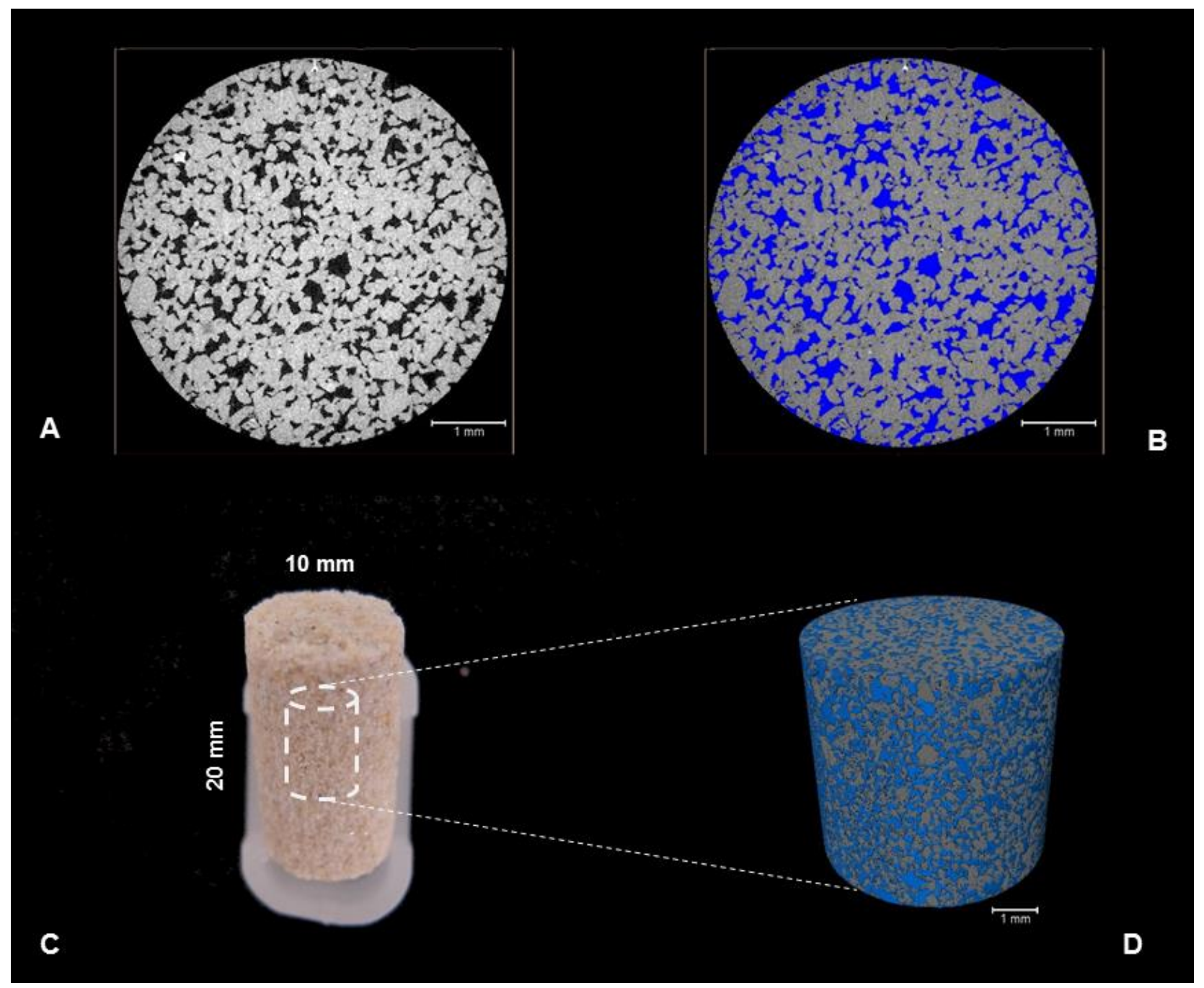

Figura 1 - BS (Bentheimer Sandstone). A - Corte 2D da imagem de MRX reconstruída. B - Segmentação 2D (azul). C - Volume de dados tratados digitalmente. D - Segmentação 3D.

A amostra BS (Bentheimer Sandstone) é um arenito homogêneo de cor amarela clara com grãos bem selecionados e moderadamente angulosos. A figura $1 \mathrm{~A}$ mostra o corte bidimensional da imagem tomográfica reconstruída, com seus minerais em tons de cinza e os espaços vazios em preto. Na figura $1 \mathrm{~B}$ os espaços vazios foram segmentados com a cor azul e posteriormente criado um modelo tridimensional (figura 1D) a partir desta segmentação. A figura 1C mostra o arenito BS (Bentheimer Sandstone) cortado em formato cilíndrico de aproximadamente $10 \mathrm{~mm}$ de diâmetro por $20 \mathrm{~mm}$ de altura e o respectivo volume selecionado para a construção do modelo tridimensional do volume de poros. A porosidade foi calculada considerando-se o volume tridimensional (figura 1D) gerado após a segmentação. A amostra apresenta um volume total

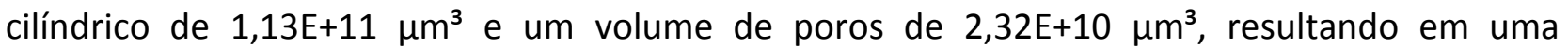
porosidade de 20,50 \%. Deste volume total de poros, 99,71 \% $\left(2,31 \mathrm{E}+10 \mu \mathrm{m}^{3}\right)$ estão conectados. $\mathrm{Na}$ análise tridimensional não se observou orientação preferencial da rede de poros. 


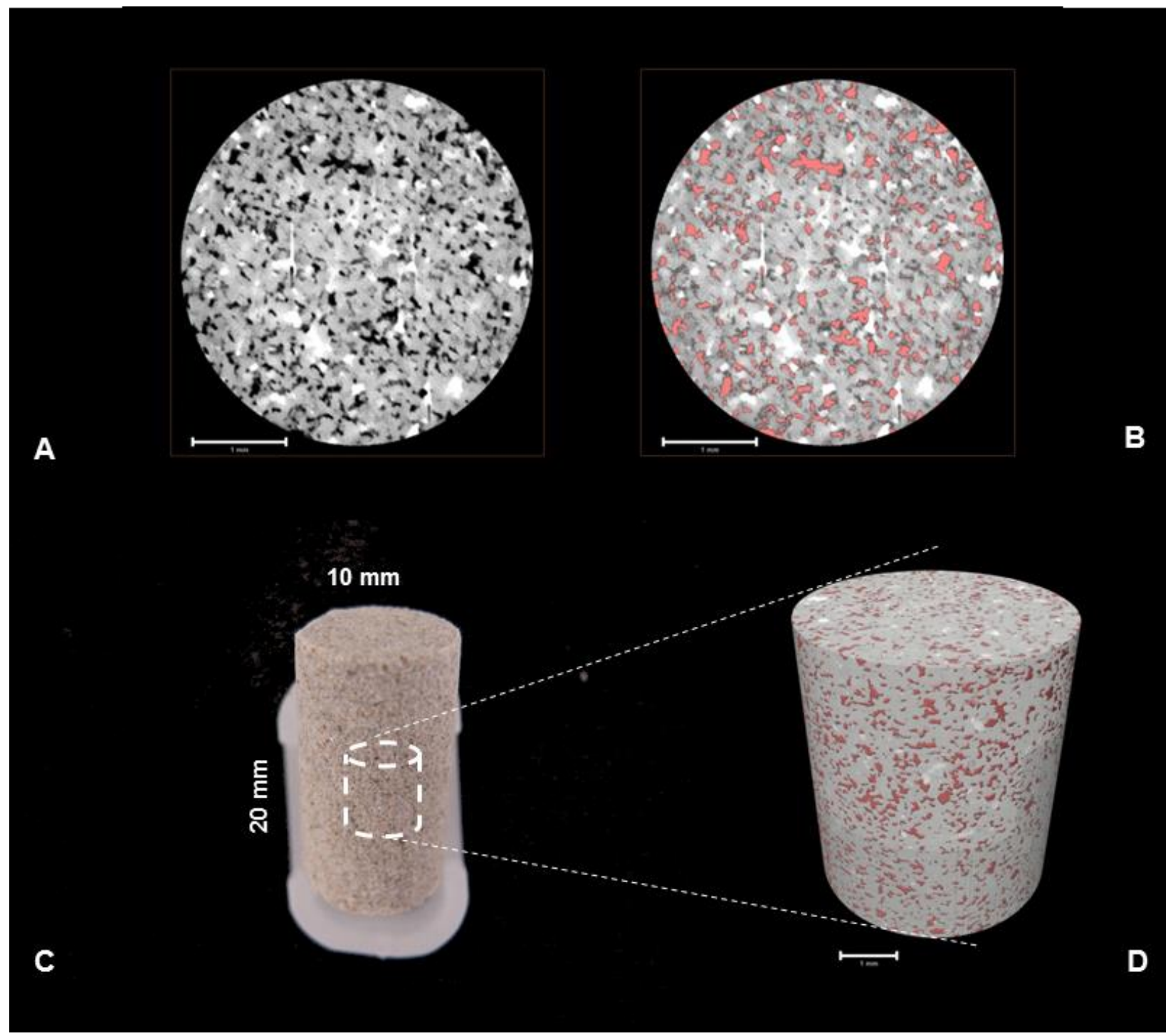

Figura 2 - GBS (Grey Berea Sandstone). A - Corte 2D da imagem de MRX reconstruída. B - Segmentação 2D (rosa claro). C - Volume de dados tratados digitalmente. D - Segmentação 3D.

A amostra GBS (Grey Berea Sandstone) é um arenito homogêneo de coloração acinzentada com grãos moderadamente selecionados e angulosos. A figura 2A mostra o corte 2D da imagem tomográfica reconstruída, com seus minerais em tons de cinza e os espaços vazios em preto. Na figura $2 \mathrm{~B}$ os espaços vazios foram segmentados com a cor rosa claro e posteriormente criado um modelo em três dimensões (figura 2D) a partir desta segmentação. Tanto a segmentação, o modelo tridimensional e a quantificação dos volumes foram realizados no software Avizo Fire da empresa FEI.

A figura 2C mostra o arenito GBS (Grey Berea Sandstone) cortado em formato de cilindro de aproximadamente $10 \mathrm{~mm}$ de diâmetro por $20 \mathrm{~mm}$ de altura e o respectivo volume selecionado para a construção do modelo tridimensional do volume de poros. A porosidade foi calculada considerando-se o volume tridimensional (figura 2D) gerado após a segmentação. A amostra apresenta um volume total cilíndrico de $6,67 \mathrm{E}+10 \mu^{3}$ e um volume de poros de $7,62 \mathrm{E}+09 \mu \mathrm{m}^{3}$, resultando em uma porosidade de $11,42 \%$. Deste volume total de poros, $84,37 \%$ $\left(6,43 \mathrm{E}+09 \mathrm{\mu m}^{3}\right)$ estão conectados. Na análise tridimensional não se observou orientação preferencial da rede de poros. 


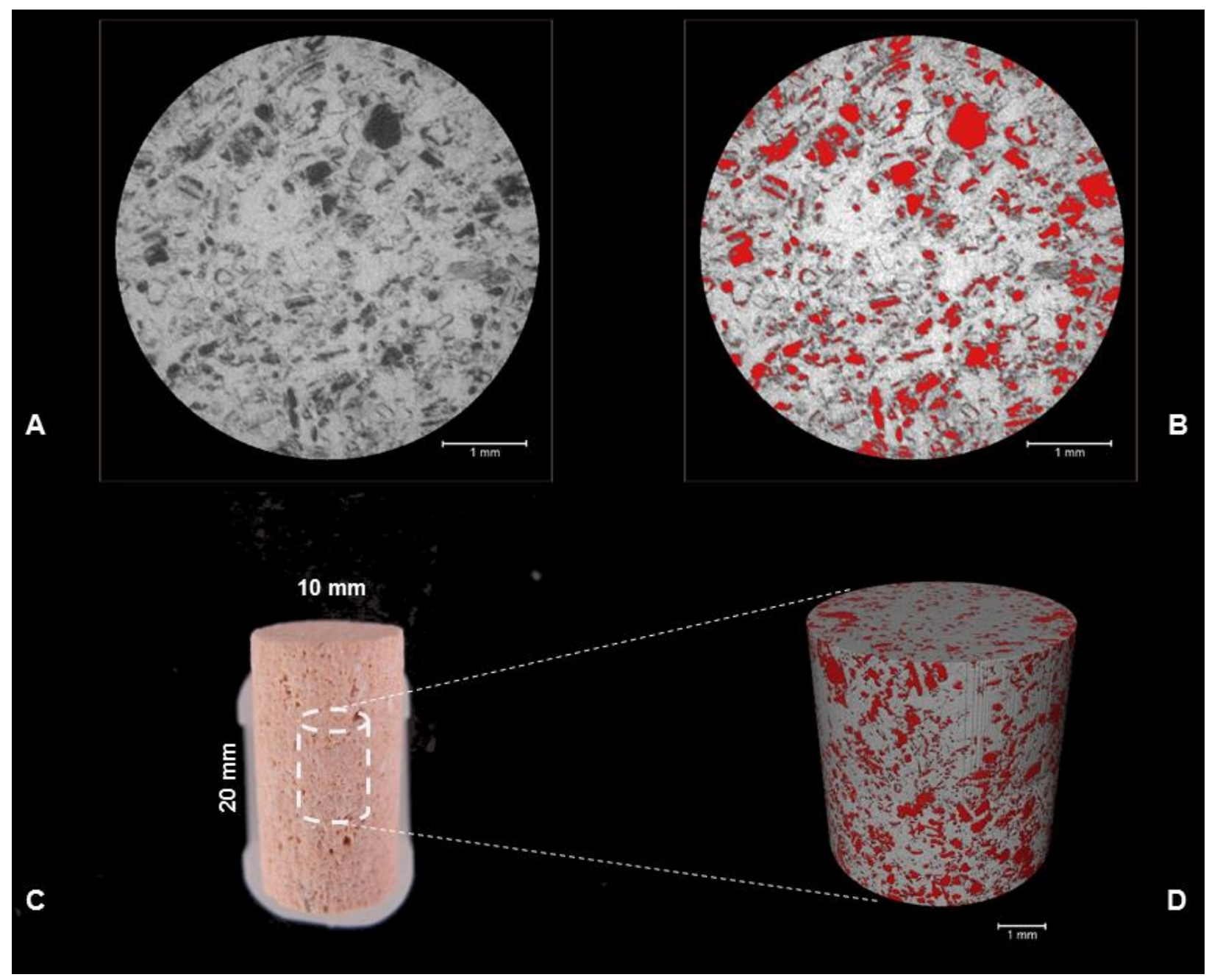

Figura 3 - DPL (Desert Pink Limestone). A - Corte 2D da imagem de MRX reconstruída. B - Segmentação 2D (vermelho). C - Volume de dados tratados digitalmente. D - Segmentação 3D.

A amostra DPL (Desert Pink Limestone) é um calcário homogêneo de coloração rosa e apresenta características isotrópicas em todo seu volume. A figura $3 \mathrm{~A}$ mostra o corte bidimensional da imagem tomográfica reconstruída, com seus minerais em tons de cinza e os espaços vazios em preto. Na figura 3B os espaços vazios foram segmentados com a cor vermelha e posteriormente criado um modelo tridimensional (figura 3D) a partir desta segmentação. Tanto a segmentação, o modelo tridimensional e a quantificação dos volumes foram realizados no software Avizo Fire da empresa FEI.

A figura 3 C mostra o calcário DPL (Desert Pink Limestone) cortado em formato cilíndrico de aproximadamente $10 \mathrm{~mm}$ de diâmetro por $20 \mathrm{~mm}$ de altura e o respectivo volume selecionado para a construção do modelo tridimensional do volume de poros. A porosidade foi calculada considerando-se o volume tridimensional (figura 3D) gerado após a segmentação. A amostra apresenta um volume total cilíndrico de $8,70 \mathrm{E}+10 \mu \mathrm{m}^{3}$ e um volume de poros de $1,65 \mathrm{E}+10 \mu \mathrm{m}^{3}$, resultando em uma porosidade de $18,97 \%$. Deste volume total de poros, $92,19 \%$ $\left(1,52 \mathrm{E}+10 \mathrm{\mu m}^{3}\right)$ estão conectados. Na análise tridimensional não se observou orientação preferencial da rede de poros. 


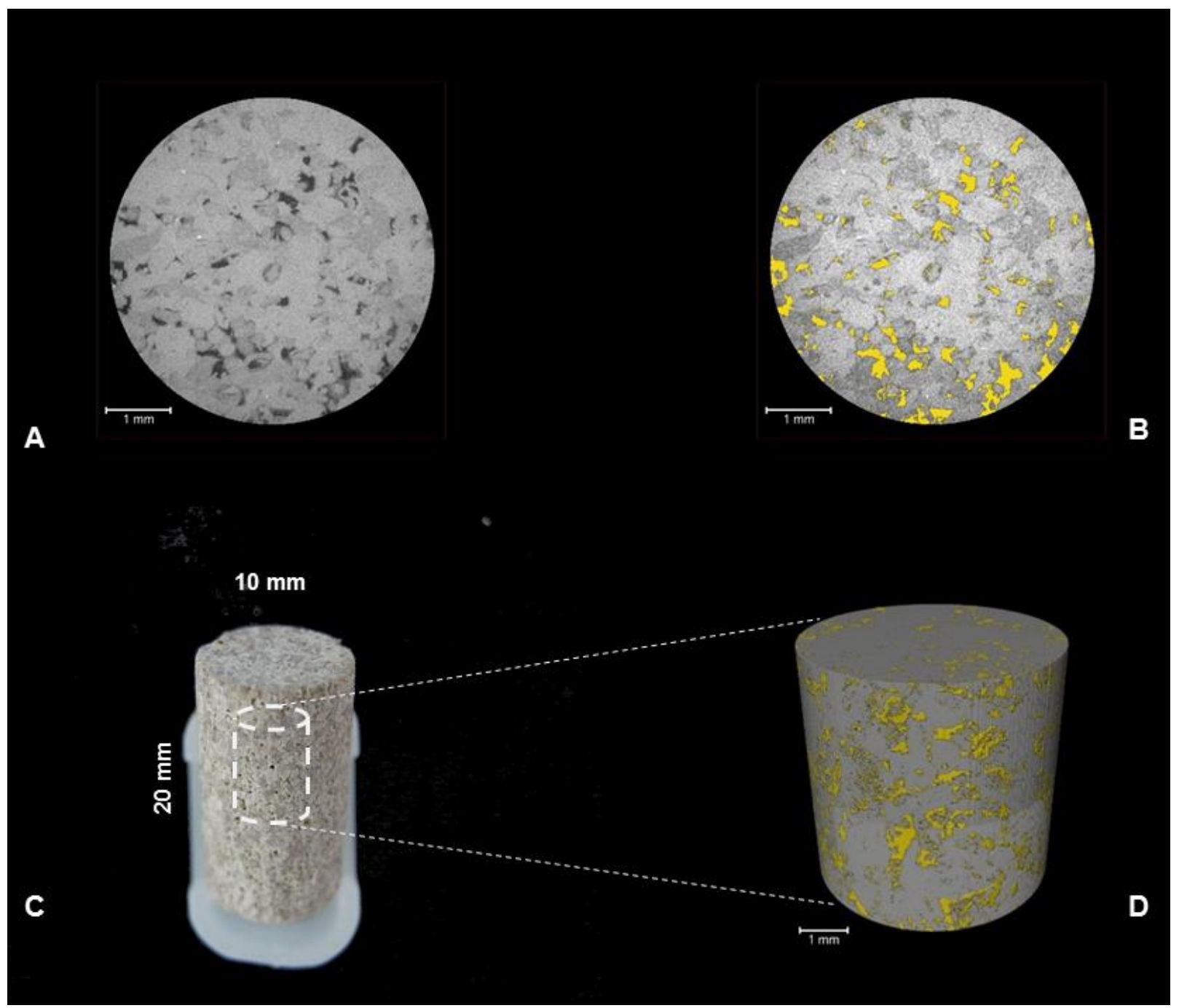

Figura 4 - IL (Indiana Limestone). A - Corte 2D da imagem de MRX reconstruída. B - Segmentação 2D (amarelo). C - Volume de dados tratados digitalmente. D - Segmentação 3D.

A amostra IL (Indiana Limestone) é um calcário homogêneo de coloração cinza e apresenta características isotrópicas em todo seu volume. A figura 4A mostra o corte bidimensional da imagem tomográfica reconstruída, com seus minerais em tons de cinza e os espaços vazios em preto. Na figura 4B os espaços vazios foram segmentados com a cor amarela e posteriormente criado um modelo tridimensional (figura 4D) a partir desta segmentação. Tanto a segmentação, o modelo tridimensional e a quantificação dos volumes foram realizados no software Avizo Fire da empresa FEl.

A figura 4C mostra o calcário IL (Indiana Limestone) cortado em formato cilíndrico de aproximadamente $10 \mathrm{~mm}$ de diâmetro por $20 \mathrm{~mm}$ de altura e o respectivo volume selecionado para a construção do modelo tridimensional do volume de poros. A porosidade foi calculada considerando-se o volume tridimensional (figura 4D) gerado após a segmentação. A amostra apresenta um volume total cilíndrico de $1,01 \mathrm{E}+11 \mu \mathrm{m}^{3}$ e um volume de poros de 1,60E+09 $\mu^{3}$, resultando em uma porosidade de 1,58\%. A amostra apresenta pouca conexão entre os poros e também não se observou orientação preferencial destes.

Os valores calculados dos volumes de poros e de volume total das quatro amostras obtidos pelas análises de MRX estão apresentados na Tabela 2. 
Tabela 2 - Dados e porosidade calculada por MRX

\begin{tabular}{crrr}
\hline Amostra & Volume de Poros $\left(\mu \mathrm{m}^{3}\right)$ & Volume Total $\left(\mu \mathrm{m}^{3}\right)$ & Porosidade MXR \\
\hline BS & $2,32 \mathrm{E}+10$ & $1,13 \mathrm{E}+11$ & $20,50 \%$ \\
GBS & $7,62 \mathrm{E}+09$ & $6,67 \mathrm{E}+10$ & $11,42 \%$ \\
DPL & $1,65 \mathrm{E}+10$ & $8,70 \mathrm{E}+10$ & $18,97 \%$ \\
IL & $1,60 \mathrm{E}+09$ & $1,01 \mathrm{E}+11$ & $1,58 \%$ \\
\hline
\end{tabular}

Os resultados são confiáveis pois apresentaram boa correlação com valores previamente estabelecidos no laboratório por porosimetria de mercúrio, que é a técnica mais consagrada para determinação de porosidade.

\section{CONCLUSÕES}

A MRX mostrou ser uma técnica simples e ágil no estudo de porosidade em laboratório, possibilitando a inspeção das microestruturas em cortes 2D não destrutivos e a quantificação de dados volumétricos como a porosidade.

Os resultados apresentados para as quatro amostras demonstram a capacidade da MRX para caracterização da estrutura de poros distintas, permitindo análises qualitativas e quantitativas de forma, volume, direção preferencial e conectividade dos poros, dados que contribuem significativamente para análise de reservatórios.

A limitação da análise de porosidade por MRX é a resolução atingida e que impossibilita a caracterização de poros submicrométricos. No entanto, para estudos de simulação de fluxo e permeabilidade, a resolução atingida $(4 \mu \mathrm{m})$ é suficiente para os modelos atuais de simulação, sendo a porosidade total determinada equivalente à porosidade efetiva das rochas reservatório.

\section{REFERÊNCIAS BIBLIOGRÁFICAS}

1. DULIU, O. G. Computer axial tomography in geosciences: an overview. Earth-Science Reviews, v. 48, n. 4, p. 265-281, Dec 1999.

2. LIN et al. Cone beam X-ray microtomography: a new facility for three-dimensional analysis of multiphase materials. Minerals \& Metallurgical Processing, v. 19, n. 2, p. 7, 2002.

3. MEES, F. et al. Applications of X-ray computed tomography in the geosciences. Geological Society, London, Special Publications, v. 215, n. 1, p. 1-6, 2003.

4. MILLER, J. D.; LIN, C. L. Three-dimensional analysis of particulates in mineral processing systems by cone beam X-ray microtomography. Minerals \& Metallurgical Processing, v. 21, n. 3, p. 113-124, 2004. 